\title{
Three-Dimensional W-Band Klystrino Simulation
}

\author{
M. Schmolke and W. Bruns \\ Technische Universität Berlin, EN2, Einsteinufer 17, D-10587 Berlin, Germany
}

Abstract

Our work is focused on the design of a small and light, simple and cheap millimeter-wave source. The present choice is a modular klystrino for medium power $(10 \ldots 50$ $\mathrm{kW}$ ) employing a $50 \mathrm{kV}$ electron beam and periodic permanent magnet (PPM) focusing. In the paper we present the results of three dimensional computer simulations for a single klystrino stage. Particle-in-cell (PIC-) simulations have been performed in order to study beam bunching and beam transport. These computations are carried out with the parallel version of GdfidL.

\section{INTRODUCTION}

The paper deals with the design of a small power source. The requirements are: Operating frequency $91.392 \mathrm{GHz}$, DC-Voltage $50 \mathrm{kV}$ and output power $5 \mathrm{~kW}$. Otherwise, all parameters are free.

We choose to design a pencil beam klystron, and not a TWT, or a Gyrotron or a magnetron or a sheet beam klystron, because:

The bandwidth of a TWT is high but the efficiency is low at millimeter wave frequencies. For the intended application a high bandwidth is not needed. Regarding the magnetron, for efficient operation the DC voltage is too high for the small gap size corresponding to W-Band. The gyrotron seems to be too complicated for our limited experience. For low $\beta$, sheet beam klystron cavities have a much lower shunt impedance than round cavities. Since we want to use a DC-voltage of only $50 \mathrm{kV}$, we can't use a sheet beam.

\section{INPUT CAVITY}

Since the dimensions of the klystrino will be in the mmregime, we want to build it with LIGA. LIGA built structures should better be planar, ie. there should not be too many different depths in the design. Therefore a rectangular input cavity is chosen. For a high Q and R/Q of the input cavity, a quadratic cross-section is used. This also has the nice effect that the modulating field is almost round in the region of the beam. With the width chosen to be equal to the depth, we adjust the depth and the gap for the operating frequency and the highest shunt-impedance for $\beta=0.413$. This $\beta$ corresponds to the chosen DC-voltage of $50 \mathrm{kV}$. For a single modulating cavity, we get a shunt-impedance of $\mathrm{R}$ $=39 \mathrm{kOhms}$. To achieve a modulating voltage of $2 \mathrm{kV}$, an input power of about 100 Watts is required. This modulating voltage corresponds to a $\gamma$ variation of $\Delta \gamma=3.9 \cdot 10^{-3}$.

\section{DC-CURRENT}

Since we have already designed a pencil beam electron gun, which is capable of delivering up to $0.48 \mathrm{Amps}$, that value is taken. The diameter of the beam also comes from that gun design and is $0.4 \mathrm{~mm}$.

\section{OUTPUT STRUCTURE, FIRST PART}

At first the output cavity is designed such that it has a high enough shunt-impedance. Assuming a loaded Q of 600 , a current modulation $m=I_{r f} / I=1.6$ and the already chosen beam-parameters $V_{0}=50 \mathrm{kV}, I=0.48$ Amps, a shunt-impedance of $R=16 \eta V_{0} /\left(m^{2} I_{0}\right)=228$ $\mathrm{kOhms}$ is required for a conversion efficiency $\eta=35 \%$. Only then we can hope for an output power of $8 \mathrm{~kW}$. From eigenvalue computations, it is clear that at least six output cavities are needed, operating in $\pi$-mode.

Analytical space charge wave calculations predict that the output cavity should be located at about $17 \mathrm{~mm}$ behind the modulation cavity.

\section{PPM-FOCUSING}

The beam can be focused in the drift region by a sinusoidal magnetic field $B_{z}=B_{0} \sin (2 \pi z / L)$. For optimum focusing the rms field value should equal the BRILLOUIN field for the beam where the magnetic focusing force just compensates space-charge- and centrifugal force [1]. According to our beam parameters the BRILLOUIN field is $B=194 \mathrm{mT}$, requiring an amplitude $B_{0}=274$ $\mathrm{mT}$. Since for stable operation, the periodicity $L$ should be small compared to the plasma wavelength $\lambda_{q}=67 \mathrm{~mm}$, we take $L=10 \mathrm{~mm}$. We realise an almost sinusoidal magnetic field near the axis with a periodic configuration of round magnets. The un-optimised magnet configuration has a ratio of third to first harmonic of 0.236 .

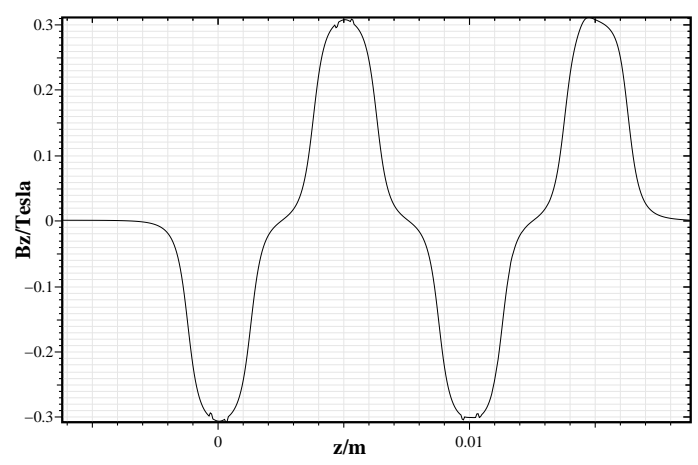

Figure 1: The z-component of the focusing field. The ratio of the third space harmonic to the first is 0.236 . 


\section{PIC-SIMULATION}

We test our paper design with GdfidLs fully 3D algorithm with free moving charges (PIC). The computational volume is $4 \mathrm{~mm} \times 4 \mathrm{~mm} \times 25 \mathrm{~mm}$, divided into 3 million cells. About 100.000 macroparticles are drifting at any time through the computational volume. A simulation of 700 RF-periods takes about 12 hours on 8 processors of an IBM SP2. GdfidL subdivides the computational volume in as many subvolumes as processors are available. Each processor advances in its subvolume the electromagnetic fields with FDTD, integrates the convection current and pushes the particles according to the LORENTZ forces.

\subsection{Position of the output cavity}

From the $\gamma$ plot of a structure without output cavities, fig. 2, we find that the analytical optimum position of the output cavity at $17 \mathrm{~mm}$ is about right. The PIC-derived position is $15 \mathrm{~mm}$.

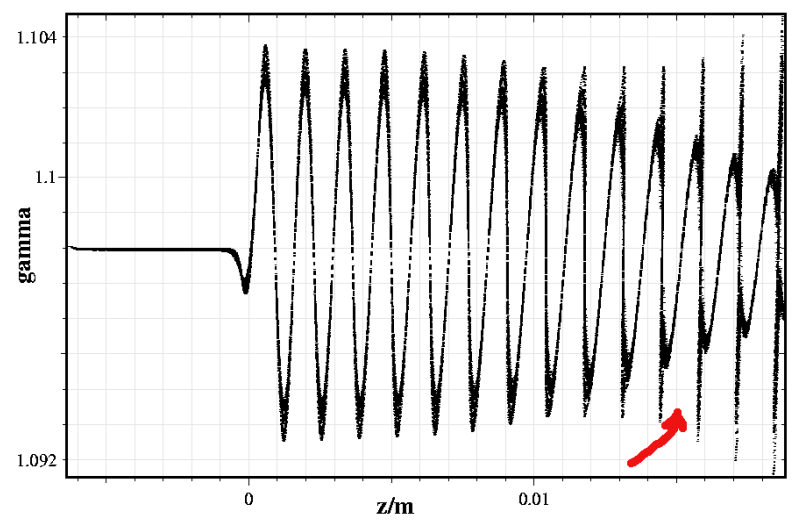

Figure 2: $\gamma$ plot in a structure with only the modulating cavity. The optimum position of the output structure seems to be near $15 \mathrm{~mm}$.

\subsection{Tapering of the output structure}

A simulation with an output structure consisting of six cavities with equal gaps, fig. 3 , shows that the velocity of the particles decreases very rapidly. It is absolutely necessary to adjust the gaps of the output cavities for synchronicity between particles and fields. The gaps are adjusted assuming a linear $\beta$ variation. The $\gamma$ distribution with the tapered output cavity is shown in fig. 4. The $\gamma$ decrease due to the output cavity is shown in fig. 5 .

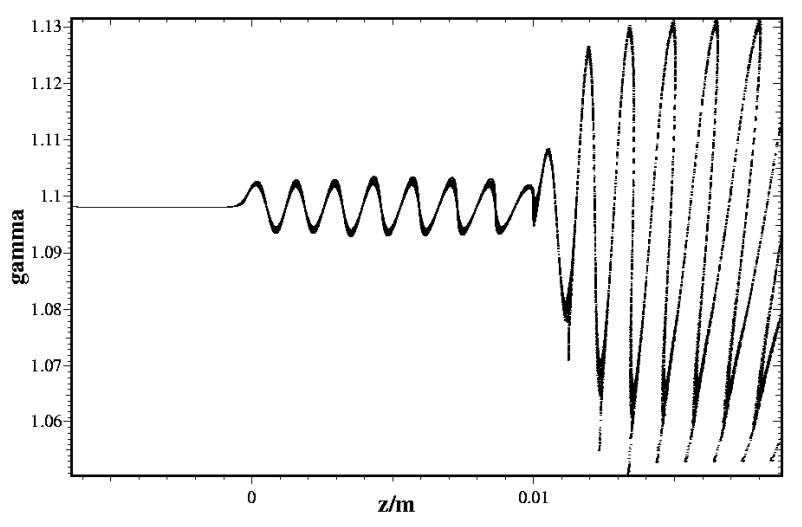

Figure 3: $\gamma$ plot in a structure with output cavity. Its first gap is located at $\mathrm{z}=10 \mathrm{~mm}$.

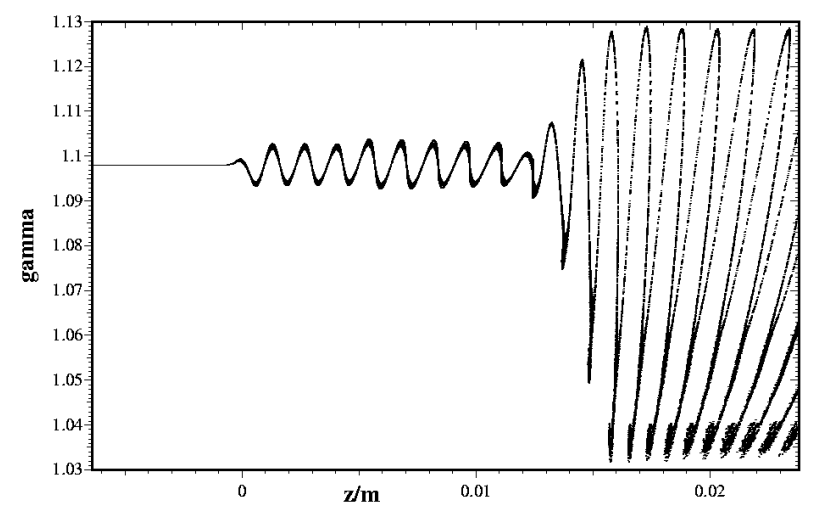

Figure 4: $\gamma$ distribution in a structure with tapered output cavity. The data is recorded after 250 periods.

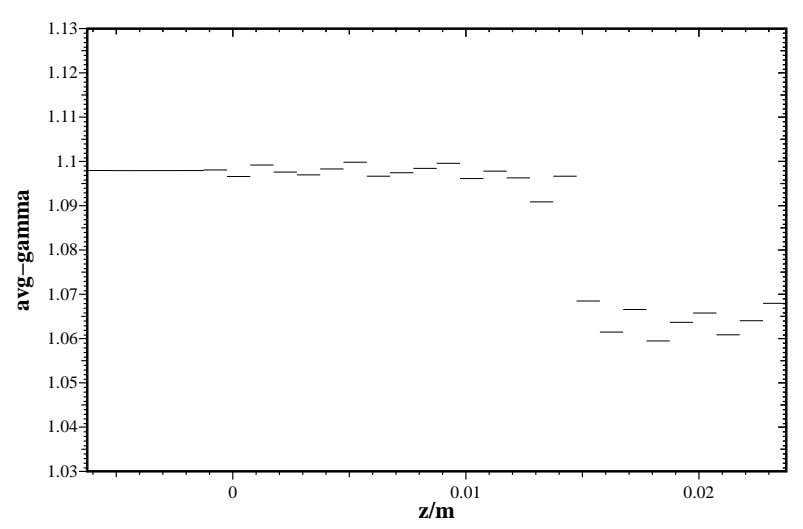

Figure 5: The average of the $\gamma$ values of fig. 4 as a function of $z$. One sees that the output cavity extracts already $30 \%$ of the kinetic energy. 


\section{OUTPUT COUPLER}

The loaded Q of the output cavity is adjusted to a value of 600 by attaching a waveguide on both sides of each of the six cells. A power combining network will be attached on both sides of the klystrino. Since both the klystrino and the network can be fabricated by LIGA, a complicated network does not cost any more than a simple one.

Figure 7 gives the power flowing through the waveguides as a function of time. Already after 130 periods, the power reaches $1 \mathrm{~kW}$.

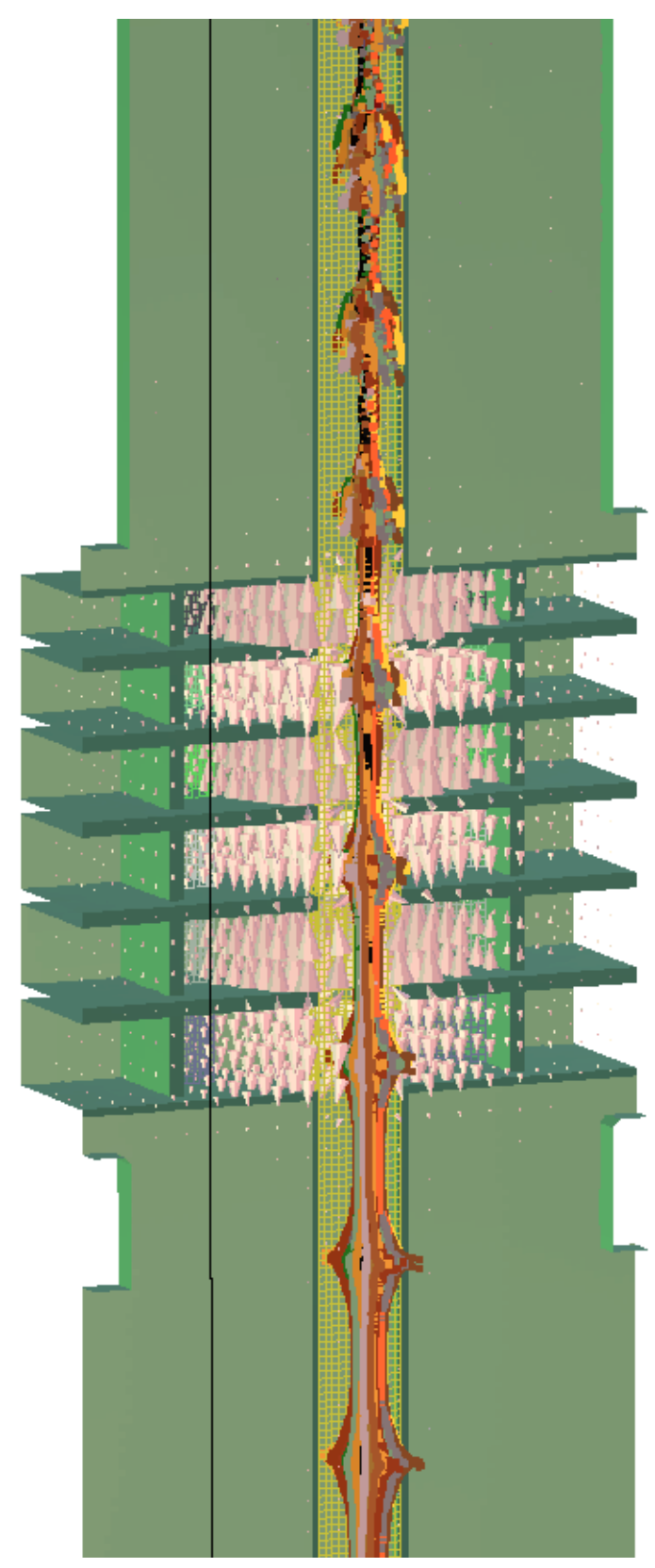

Figure 6: Macroparticles and electric field in the output cavity with attached coupler after 500 periods. The different colours of the macroparticles do not indicate different species. They are used for identifying the particles being emitted at the same transversal positions.

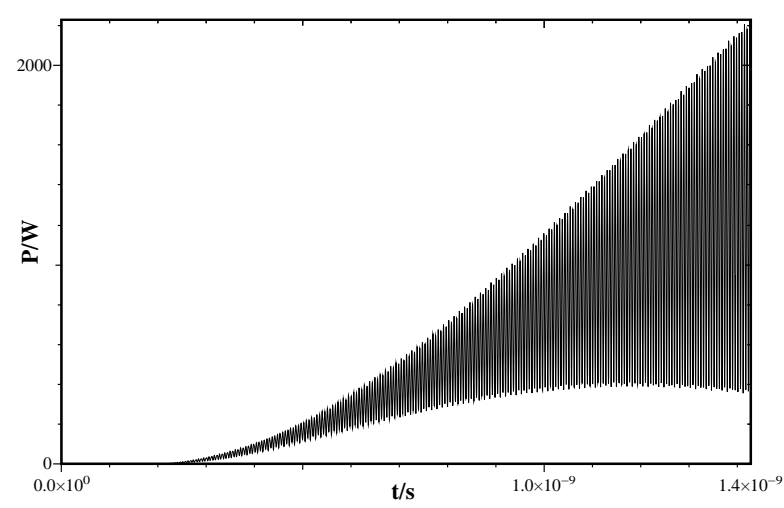

Figure 7: The extracted power as a function of time for the first 130 periods. The lower margin of the envelope is the power in higher harmonics. The upper margin is the total power in all harmonics. The average power after 130 periods is about $1 \mathrm{~kW}$.

\section{CONCLUSION}

Analytical formulas have been used for designing a W-Band klystrino. The electromagnetic field simulator GdfidL has been used to test that first design. The agreement between the analytical expectations and the numerical results is satisfactory. Further work is needed to increase the efficiency. The PPM-focusing works good. Almost no particles hit the beam-pipe.

\section{REFERENCES}

[1] J.T. Mendel, C.F. Quate, W.H. Yocom, "Electron Beam Focusing with Periodic Permanent Magnet Fields", Proc. IRE, pp. 800-810 (May 1954)

[2] Robert E. Collin, "Foundations for Microwave Engineering", McGraw-Hill, 2nd ed. (1992)

[3] A. S. Gilmour, "Principles of traveling wave tubes", Artech House, (1994)

[4] "The GdfidL Electromagnetic Field Simulator", http://www.wbfb.de 